\title{
Tidal acceleration of ultrarelativistic particles
}

\author{
C. Chicone ${ }^{1}$ and B. Mashhoon ${ }^{2}$ \\ 1 Department of Mathematics, University of Missouri-Columbia, Columbia, Missouri 65211, USA \\ 2 Department of Physics and Astronomy, University of Missouri-Columbia, Columbia, Missouri 65211, USA \\ e-mail: MashhoonB@missouri .edu
}

Received 29 March 2005 / Accepted 27 May 2005

\begin{abstract}
We investigate the motion of free relativistic particles relative to the ambient medium around a gravitationally collapsed system. If the relative speed exceeds a critical value given by $c / \sqrt{2}$, the gravitational tidal effects exhibit novel features that are contrary to Newtonian expectations. In particular, ultrarelativistic jet clumps moving freely outward along the rotation axis strongly decelerate with respect to the ambient medium, while ultrarelativistic particles strongly accelerate in directions normal to the jet axis. The implications of these direct consequences of general relativity for jets in microquasars and the origin of the high-energy cosmic rays are briefly mentioned.
\end{abstract}

Key words. gravitation - acceleration of particles - black hole physics

\section{Introduction}

The purpose of this Letter is to discuss some of the observable consequences of the motion of relativistic particles in the field of gravitationally collapsed configurations. In particular, we point out novel features of general relativity regarding the tidal influence of a neutron star or a black hole on the motion of nearby free particles moving faster than the critical speed $c / \sqrt{2} \approx 0.7 c$ relative to the ambient medium surrounding the central mass. Such ultrarelativistic particles originating near the poles of the collapsed system suffer significant tidal $d e$ celeration within a cone of angle $\theta \approx 55^{\circ}$ around the axis of rotation. Here $\theta$ represents half the angle at the vertex of the cone. Outside the cone, however, an ultrarelativistic particle experiences tidal acceleration. The situation is depicted schematically in Fig. 1 for the north pole of a Kerr black hole. Similar outflows are expected near the south pole.

The solutions of the geodesic equation as well as the Lorentz force equation in black hole fields in standard Schwarzschild-like systems of coordinates have been extensively studied and many useful results have thereby been obtained (see, e.g., Chandrasekhar 1983). For the study of certain phenomena, however, it is more useful to study relative motion, i.e. motion of one particle with respect to another. This is in keeping with the spirit of the theory of relativity. It also corresponds to the physical interpretation of some astronomical situations. Consider, for instance, the Chandra $\mathrm{X}$-ray images of the Crab Nebula: to reveal the accelerating rings in the equatorial plane, different images have to be compared with each other keeping certain central features "fixed" (http://chandra.harvard. edu/photo/2002/0052/ index.html). To study relative motion in an invariant setting

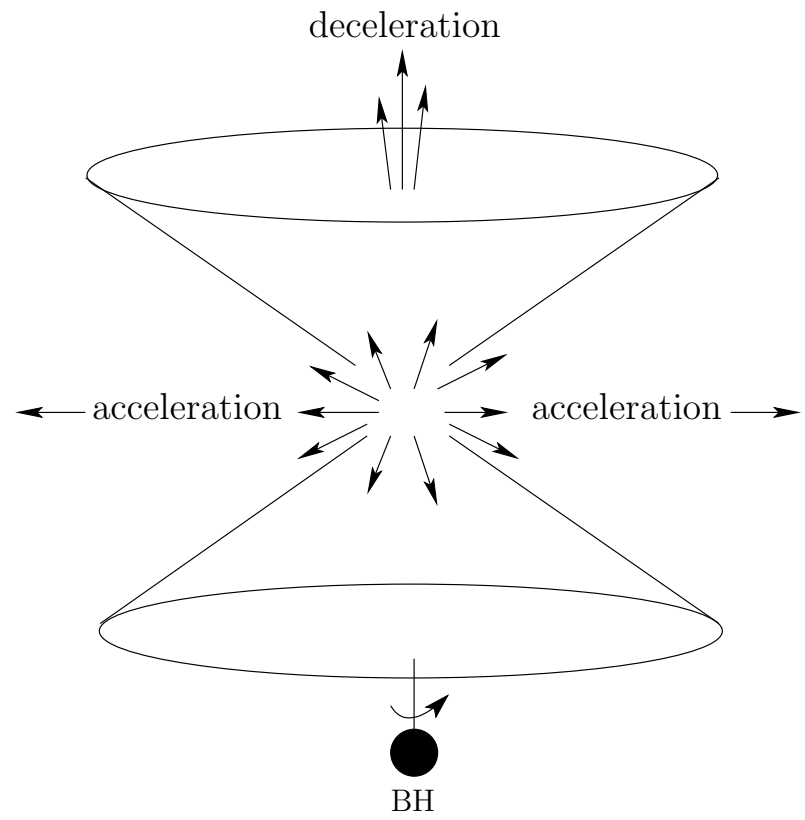

Fig. 1. Schematic diagram of the critical velocity cone.

corresponding to actual observations, it is useful to establish a Fermi normal coordinate system along a reference geodesic $O$. The motion of a nearby free particle with respect to $O$ is then given by the geodesic equation in the quasi-inertial Fermi coordinate system (Synge 1960).

Let $\tau$ be the proper time and $\lambda^{\mu}{ }_{(0)}=\mathrm{d} x^{\mu} / \mathrm{d} \tau$ be the fourvelocity vector of $O$. A triad of ideal orthonormal gyro directions $\lambda_{(i)}^{\mu}, i=1,2,3$, can be parallel propagated along $O$ 's worldline such that $\lambda_{(\alpha)}^{\mu}(\tau)$ is a local orthonormal tetrad frame. 
Based on these local axes, the Fermi coordinates $(T, X)$ simply provide a geodesic normal coordinate system along the worldline of $O$. The reference worldline is then the temporal axis ( $T=\tau, \boldsymbol{X}=\mathbf{0}$ ) and the metric tensor in the Fermi system is given by

$$
\begin{aligned}
& g_{00}=-1-{ }^{F} R_{0 i 0 j}(T) X^{i} X^{j}+\cdots, \\
& g_{0 i}=-\frac{2}{3}{ }^{F} R_{0 j i k}(T) X^{j} X^{k}+\cdots, \\
& g_{i j}=\delta_{i j}-\frac{1}{3}{ }^{F} R_{i k j \ell}(T) X^{k} X^{\ell}+\cdots,
\end{aligned}
$$

where

$$
{ }^{F} R_{\alpha \beta \gamma \delta}(T)=R_{\mu \nu \rho \sigma} \lambda_{(\alpha)}^{\mu} \lambda_{(\beta)}^{v} \lambda_{(\gamma)}^{\rho} \lambda_{(\delta)}^{\sigma}
$$

is the projection of the Riemann tensor on the local frame of $O$. Along $O$ 's worldline, the metric reduces to the Minkowski metric according to Eq. (1). The deviations from an inertial system of coordinates are quadratic and higher order in distance away from the reference worldline. The Fermi coordinates are admissible only in a cylindrical region of radius $\mathcal{R}(T)$ around the reference worldline, where $\mathcal{R}$ is a certain minimum radius of curvature determined via the Riemann tensor and its covariant derivatives along the worldline of $O$. The equations of general relativity may be expressed in any admissible coordinate system. We are interested in the geodesic equation of motion in the quasi-inertial Fermi coordinate patch.

\section{Generalized Jacobi equation}

The geodesic equation for a free test particle $\mathcal{P}$ in the Fermi system can be reduced to the general tidal equation (Mashhoon 1975, 1977); however, if we limit our considerations to the terms given explicitly in Eq. (1), we get the generalized Jacobi equation (Chicone \& Mashhoon 2002)

$$
\begin{aligned}
& \frac{\mathrm{d}^{2} X^{i}}{\mathrm{~d} T^{2}}+{ }^{F} R_{0 i 0 j} X^{j}+2{ }^{F} R_{i k j 0} V^{k} X^{j} \\
& +\frac{2}{3}\left(3{ }^{F} R_{0 k j 0} V^{i} V^{k}+{ }^{F} R_{i k j \ell} V^{k} V^{\ell}+{ }^{F} R_{0 k j \ell} V^{i} V^{k} V^{\ell}\right) X^{j}=0,
\end{aligned}
$$

where $\boldsymbol{V}=\mathrm{d} \boldsymbol{X} / \mathrm{d} T$ is such that the four-velocity of $\mathcal{P}$ is given by $U^{\mu}=\Gamma(1, \boldsymbol{V})$ and $\Gamma$ is the modified Lorentz factor given by

$$
\begin{aligned}
\frac{1}{\Gamma^{2}}= & 1-V^{2}+{ }^{F} R_{0 i 0 j} X^{i} X^{j}+\frac{4}{3}{ }^{F} R_{0 j i k} X^{j} V^{i} X^{k} \\
& +\frac{1}{3}{ }^{F} R_{i k j \ell} V^{i} X^{k} V^{j} X^{\ell}
\end{aligned}
$$

at the level of approximation under consideration here. For $|\boldsymbol{V}| \ll 1$, we may neglect all terms proportional to the relative velocity in the equations of motion and Eq. (3) reduces to the familiar Jacobi equation (Synge 1960)

$$
\frac{\mathrm{d}^{2} X^{i}}{\mathrm{~d} T^{2}}+K_{i j} X^{j}=0
$$

Here $K_{i j}={ }^{F} R_{0 i 0 j}$ is the symmetric tidal matrix that reduces in the Newtonian limit to $\partial^{2} \Phi / \partial X^{i} \partial X^{j}$, where $\Phi$ is the Newtonian gravitational potential. The Jacobi equation expresses the linear evolution of the deviation between two neighboring geodesic worldlines with negligible relative motion. Equation (5) has been employed extensively in the treatment of tidal effects in general relativity (e.g. Stewart \& Walker 1973; Mashhoon 1975, 1977; Carter \& Luminet 1985; Ivanov \& Novikov 2001). Detailed calculations have revealed that the dominant tidal effects of massive bodies are rather similar to their Newtonian counterparts except possibly very close to black holes.

Equation (4) follows from the timelike character of particle motion; that is, the requirement that $g_{\mu \nu} U^{\mu} U^{\nu}=-1$ leads to Eq. (4) when we use the terms in the expansion of the metric tensor given explicitly in Eq. (1). The modified Lorentz factor is positive and approaches infinity if the timelike geodesic approaches a null geodesic. It follows from Eq. (4) that $V^{2}<1$ along the reference geodesic; elsewhere in the Fermi frame, however, $V^{2}$ is simply constrained by the requirement that the right-hand side of Eq. (4) be positive.

The quasi-inertial Fermi coordinate system has been widely used in the theory of general relativity; for example, it is employed by Ehlers \& Rindler (1997) to study the local bending of light in a gravitational field.

\section{Tidal acceleration in Kerr spacetime}

In this Letter we point out the major consequences of Eqs. (3) and (4) for ultrarelativistic motion in the field of a rotating source represented by the exterior Kerr spacetime. The Kerr metric can be expressed in Boyer-Lindquist coordinates $(t, r, \vartheta, \phi)$ as

$$
\begin{aligned}
\mathrm{d} s^{2}= & -\mathrm{d} t^{2}+\Sigma\left(\frac{1}{\Delta} \mathrm{d} r^{2}+\mathrm{d} \vartheta^{2}\right)+\left(r^{2}+a^{2}\right) \sin ^{2} \vartheta \mathrm{d} \phi^{2} \\
& +2 G M \frac{r}{\Sigma}\left(\mathrm{d} t-a \sin ^{2} \vartheta \mathrm{d} \phi\right)^{2}
\end{aligned}
$$

where $\Sigma=r^{2}+a^{2} \cos ^{2} \vartheta$ and $\Delta=r^{2}-2 G M r+a^{2}$. Here $M$ is the mass, $J=M a$ is the angular momentum of the source and we use units such that $c=1$. We take $O$ to be a test particle moving along the axis of rotation $(\vartheta=0)$ on an escape trajectory such that it reaches infinity with a speed that is small compared to unity; in fact, we set this speed equal to zero, since our results turn out to be insensitive to it. The worldline of the reference particle is thus characterized by

$$
\frac{\mathrm{d} t}{\mathrm{~d} \tau}=\frac{r^{2}+a^{2}}{r^{2}-2 G M r+a^{2}}, \quad \frac{\mathrm{d} r}{\mathrm{~d} \tau}=\sqrt{\frac{2 G M r}{r^{2}+a^{2}}} .
$$

We assume that at $t=\tau=0$, the reference particle starts from $r=r_{0}>\sqrt{3} a$ along the rotation axis and eventually escapes to infinity in the absence of other interactions. Given this reference trajectory, it is then necessary to choose the spatial axes of the tetrad frame $\lambda^{\mu}{ }_{(\alpha)}$. Taking $\lambda^{\mu}{ }_{(3)}$ to be along the direction of motion, axial symmetry about this direction implies that there is rotational degeneracy in the choice of $\lambda_{(1)}^{\mu}$ and $\lambda_{(2)}^{\mu}$. Once a triad is chosen, however, it is parallel propagated along the reference worldline. The projection of the Riemann tensor on this tetrad, as in Eq. (2), is given by a symmetric $6 \times 6$ matrix with indices that range over the set $\{01,02,03,23,31,12\}$ :

$$
\left[\begin{array}{cc}
E & B \\
B & -E
\end{array}\right]
$$


where $E$ and $B$ are symmetric and traceless $3 \times 3$ matrices that represent the electric and magnetic parts of the curvature tensor. It turns out (Mashhoon et al. 1999) that $E$ and $B$ are both diagonal and parallel: $E / k=B / q=\operatorname{diag}\left(-\frac{1}{2},-\frac{1}{2}, 1\right)$, where

$k=-2 G M \frac{r\left(r^{2}-3 a^{2}\right)}{\left(r^{2}+a^{2}\right)^{3}}, \quad q=2 G M a \frac{3 r^{2}-a^{2}}{\left(r^{2}+a^{2}\right)^{3}}$.

Integrating the equation of motion of the reference geodesic Eq. (7), we find $r(T)$ and its substitution in Eq. (9) gives the electric and magnetic curvatures $k(T)<0$ and $q(T)>0$, respectively. The class of such reference particles provides an ambient medium around the source. In effect, this medium provides a local dynamic connection with the rest frame of the source.

The generalized Jacobi Eq. (3) in the case under consideration takes the form

$$
\begin{aligned}
\ddot{X}- & \frac{1}{2} k X\left[1-2 \dot{X}^{2}+\frac{2}{3}\left(2 \dot{Y}^{2}-\dot{Z}^{2}\right)\right]+\frac{1}{3} k \dot{X}(5 Y \dot{Y}-7 Z \dot{Z}) \\
& +q\left[\dot{X} \dot{Y} \dot{Z} X-\dot{Z} Y\left(1+\dot{X}^{2}\right)-2 \dot{Y} Z\right]=0, \\
\ddot{Y}- & \frac{1}{2} k Y\left[1-2 \dot{Y}^{2}+\frac{2}{3}\left(2 \dot{X}^{2}-\dot{Z}^{2}\right)\right]+\frac{1}{3} k \dot{Y}(5 X \dot{X}-7 Z \dot{Z}) \\
& \quad-q\left[\dot{X} \dot{Y} \dot{Z} Y-\dot{Z} X\left(1+\dot{Y}^{2}\right)-2 \dot{X} Z\right]=0, \\
\ddot{Z}+ & k Z\left[1-2 \dot{Z}^{2}+\frac{1}{3}\left(\dot{X}^{2}+\dot{Y}^{2}\right)\right]+\frac{2}{3} k \dot{Z}(X \dot{X}+Y \dot{Y}) \\
& -q(X \dot{Y}-\dot{X} Y)\left(1-\dot{Z}^{2}\right)=0,
\end{aligned}
$$

where $\dot{X}=\mathrm{d} X / \mathrm{d} T$, etc. Let us first concentrate on the motion of $\mathcal{P}$ along the rotation axis; that is, $X(T)=Y(T)=0$ for all $T \geq 0$. In this case, Eqs. (10)-(12) reduce to

$$
\frac{\mathrm{d}^{2} Z}{\mathrm{~d} T^{2}}+k\left(1-2 \dot{Z}^{2}\right) Z=0 \text {. }
$$

The character of the solutions of this equation depends drastically on whether the relative initial velocity along the $Z$-direction has magnitude below or above the critical speed $1 / \sqrt{2}$. For initially infrarelativistic motion (i.e. $\dot{Z}^{2}<1 / 2$ ), Eq. (13) implies that the particle accelerates until it asymptotically approaches the critical speed. For initially ultrarelativistic motion (i.e. $\dot{Z}^{2}>1 / 2$ ), however, the particle strongly decelerates regardless of its initial Lorentz factor $\Gamma_{0}>\sqrt{2}$ and asymptotically approaches the critical speed $1 / \sqrt{2}$. This deceleration phenomenon is consistent with the observations of Galactic "superluminal" jets (Fender 2004).

Let us next consider motion along the $X$-axis; that is, $Y(T)=Z(T)=0$ for all $T \geq 0$. Then Eqs. (10)-(12) reduce to

$\frac{\mathrm{d}^{2} X}{\mathrm{~d} T^{2}}-\frac{1}{2} k\left(1-2 \dot{X}^{2}\right) X=0$,

which turns out to be a generic equation for motion perpendicular to the symmetry axis as a consequence of the rotational symmetry of Eqs. (10)-(12) about the Z-axis. For initially infrarelativistic motion, it follows from Eq. (14) that the particle slowly decelerates, while for initially ultrarelativistic motion, the particle strongly accelerates as depicted in Fig. 2 . The latter situation is remarkable, as it can lead to $\Gamma \rightarrow \infty$ in the absence of other interactions. It is clear from Fig. 2 that in some cases the Fermi coordinate system could break down before $\Gamma \rightarrow \infty$.

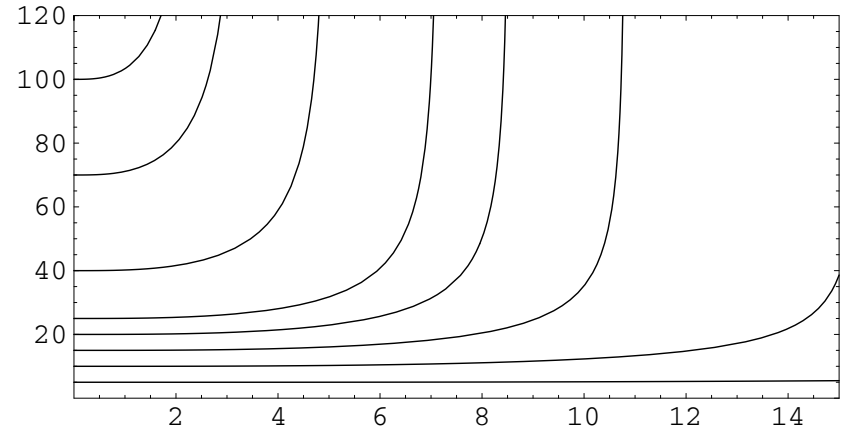

Fig. 2. Plot of the Lorentz factor $\Gamma=\left(1-\dot{X}^{2}-k X^{2} / 2\right)^{-1 / 2}$ versus $T /(G M)$ based on integration of Eq. (14) with initial data $X=0$ at $T=$ 0 with $\dot{X}(0)=\left(\Gamma_{0}^{2}-1\right)^{1 / 2} / \Gamma_{0}$ corresponding respectively to $\Gamma_{0}=5,10$, $15,20,25,40,70$ and 100 . In this plot $a /(G M)=1$ and $r_{0} /(G M)=15$. The graph illustrates acceleration of the particle such that $\Gamma$ essentially approaches infinity at $T /(G M) \approx 11,9,7,5,3$ and 2.6 for $\Gamma_{0}=15$, $20,25,40,70$ and 100, respectively.

The breakdown in our dynamics for $\Gamma \rightarrow \infty$ is logically distinct from the kinematic breakdown of the Fermi coordinate system beyond $|\boldsymbol{X}| \sim \mathcal{R}$. These tentative conclusions follow from our simple scenario that takes into account only the quadratic terms in Eq. (1); in principle, the whole infinite series must be considered. That is, the geodesic equation of motion of $\mathcal{P}$ involves an infinite series of tidal terms; the breakdown occurs when instead of the infinite set of tidal terms only the first-order terms are taken into account. Our analytical and numerical studies of the general structure of this infinite series have led us to the working hypothesis that for ultrarelativistic particles, finite but significant tidal acceleration takes place normal to the jet direction (Chicone \& Mashhoon 2005).

The breakdown of our simple theoretical scheme as $\Gamma \rightarrow \infty$ implies that the influence of such a particle on the background spacetime cannot be neglected. A more complete treatment should take this influence into account, thereby moderating the singularity that we have encountered. Moreover, the tidal energy exchange resulting in the deceleration/acceleration phenomena must be augmented to take due account of gravitational radiation energy emitted by the particles. This energy, as well as the corresponding electromagnetic radiation energy emitted by the electrically charged particles, is in the form of long-wavelength radiation of frequency proportional to $(2 G M)^{-1}=10^{5}\left(M_{\odot} / M\right) \mathrm{Hz}$. The tidal acceleration of charged particles could be strongly affected by the electromagnetic field configuration around the source.

It is interesting to consider the exchange of energy between the particle $\mathcal{P}$ and the gravitational field. Einstein's principle of equivalence prevents a local (i.e. pointwise) description of gravitational energy. From the standpoint of observers at rest with the ambient medium, however, an initially ultrarelativistic particle would lose energy to the gravitational field along the jet direction, but would gain tidal energy propagating outward in the equatorial plane; thus, the gain in gravitational energy by the accelerating particles could be essentially balanced with the loss of gravitational energy by the particles decelerating along the jets. 
For an invariant characterization of these tidal phenomena, consider the class of fundamental observers $\mathcal{S}$ that are at rest with four-velocity $u^{\mu}=(W, 0,0,0)$ with respect to the Fermi coordinate system. Here $W=\left(-g_{00}\right)^{-1 / 2}$. Let $\hat{\Gamma}:=-u^{\mu} U_{\mu}$ be the Lorentz factor of $\mathcal{P}$ measured by the static observers $\mathcal{S}$, which are in general accelerated. It turns out that

$\hat{\Gamma}=\left(W^{-1}-W g_{0 i} V^{i}\right) \Gamma$.

We find that $\hat{\Gamma}$ behaves essentially as $\Gamma$; e.g., in Fig. 2, the plot of $\hat{\Gamma}$ would be indistinguishable from that of $\Gamma$.

Ultrarelativistic particles are expected to be produced near a highly magnetized rapidly rotating neutron star or as a consequence of the complicated accretion phenomena in the vicinity of an active black hole (Punsly 2001; Guthmann et al. 2002). For our purposes, we imagine an abundance of such particles near the poles of the collapsed system. Extensive numerical studies of Eqs. (10)-(12) indicate that the deceleration along the rotation axis extends to a cone with an opening half-angle $\theta$ given by $\tan \theta \approx \sqrt{2}$; moreover, motion outside the cone corresponds to tidal acceleration away from the collapsed system.

Tidal acceleration of ultrarelativistic particles is maximum in the equatorial plane and decreases away from it, turning to deceleration at an inclination angle of about $35^{\circ}$. Our results regarding tidal acceleration appear to be consistent with recent Chandra X-ray studies of four neutron stars: Crab Pulsar, Vela Pulsar, PSR B1509-58 and SNR G54.1+0.3 (http://chandra.harvard.edu/photo/ chronological.html). In each case, there is considerable activity near the equatorial plane of the central source. Moreover, a detailed analysis of the Chandra X-ray images of the Crab Nebula suggests that the equatorial activity is somewhat more energetic than the activity along the jets (Mori et al. 2004), which is consistent with the tidal acceleration/deceleration of ultrarelativistic particles studied in this work. The same appears to be the case for the Vela Pulsar (http://chandra . harvard . edu/photo/2000/vela/ index.html).

\section{Discussion}

It is important to emphasize that the deceleration/acceleration phenomena are generic to collapsed configurations; the rotation of the central source has a negligible influence on these results. In particular, the results presented in Fig. 2 are insensitive to the assumption that the two particles initially coincide, or that the central source rotates rapidly. Numerical experiments for initially nearby particles as well as for $a$ in the range $[0, G M]$ demonstrate that the results presented here are robust. What matters is that the central source is a neutron star or a black hole; the largest effects are expected to occur in the latter case. In either case, tidal deceleration/acceleration can be significant near the source, where the ultrarelativistic particles are launched; however, a complete treatment should take due account of the complicated plasma processes as well (Punsly 2001; Guthmann et al. 2002).

The deceleration/acceleration phenomena occur relative to the ambient medium. That is, the geodesic orbits of the exterior
Kerr spacetime under consideration in this work would not appear to have any extraordinary features when considered from the standpoint of the static inertial observers at spatial infinity. The situation is different, however, in the Fermi system.

The ultrarelativistic particles that are created near a gravitationally collapsed system or in the accretion process around the system are tidally decelerated in a cone around the rotation axis of the collapsed system and appear as confined relativistic outflows from the system. These correspond to jets from some neutron stars (http: //chandra. harvard. edu/photo/ chronological.html) or X-ray binaries (microquasars) in our galaxy that have been extensively studied (Guthmann et al. 2002; Fender 2004). On the other hand, the extremely relativistic particles that result from tidal acceleration outside the cone may interact with the ambient medium within the Fermi system thereby transferring their tidal energy to the ambient particles that may escape from the system altogether and can appear as extremely energetic cosmic rays. Such neutron star or microquasar particles may account for the inferred ultrahigh energy primary cosmic rays with energies above $10^{20} \mathrm{eV}$, since the corresponding extragalactic particles would collide with the cosmic microwave background photons resulting in photopion production and pair creation (Greisen 1966; Zatsepin \& Kuzmin 1966). It would therefore be interesting to search for any correlation between the directionality associated with the highest energy cosmic rays and the distribution of certain neutron stars and microquasars in our galaxy using the Pierre Auger Observatory.

\section{References}

Carter, B., \& Luminet, J.-P. 1985, MNRAS, 212, 23

Chandrasekhar, S. 1983, The Mathematical Theory of Black Holes (New York: Oxford University Press)

Chicone, C., \& Mashhoon, B. 2002, Class. Quantum Grav., 19, 4231

Chicone, C., \& Mashhoon, B. 2005 [arXiv: astro-ph/0502560]

Ehlers, J., \& Rindler, W. 1997, Gen. Rel. Grav., 29, 519

Fender, R. 2004, in Compact Stellar X-ray Sources, ed. W. H. G. Lewin, \& M. van der Klis (Cambridge: Cambridge University Press)

Greisen, K. 1966, Phys. Rev. Lett., 16, 748

Guthmann, A. W., Georganopoulos, M., Marcowith, A., \& Manolaku, K. (eds.) 2002, Relativistic Flows in Astrophysics, Lect. Notes Phys., 589 (Berlin: Springer)

Ivanov, P. B., \& Novikov, I. D. 2001, ApJ, 549, 467

Mashhoon, B. 1975, ApJ, 197, 705

Mashhoon, B. 1977, ApJ, 216, 591

Mashhoon, B., McClune, J. C., \& Quevedo, H. 1999, Class. Quantum Grav., 16, 1137

Mori, K., Burrows, D. N., Hester, J. J., et al. 2004, ApJ, 609, 186

Punsly, B. 2001, Black Hole Gravitohydromagnetics (New York: Springer-Verlag)

Stewart, J., \& Walker, M. 1973, Commun. Math. Phys., 29, 43

Synge, J. L. 1960, Relativity: The General Theory (Amsterdam: North-Holland)

Zatsepin, G. T., \& Kuzmin, V. A. 1966, JETP Lett., 4, 78 (1966, Pis'ma Zh. Eksp. Teor. Fiz., 4, 114) 